\title{
Offsetting the bright sources for LAMOST
}

\section{Ya-Juan Lei, Hao-Tong Zhang, Yi-Qiao Dong and Hai-Long Yuan}

National Astronomical Observatories, Chinese Academy of Sciences, Beijing, China email: leiyj@lamost.org

\begin{abstract}
For optical fiber spectrum of LAMOST, the requirement for magnitude difference between neighbor fibers is less than 5 magnitudes to avoid faint sources being contaminated by their bright neighbors. However, sometime, neither bright nor faint sources are dense enough to fill a whole plate (4000 fibers). To make full use of all the fibers, they have better be put into the same plate. To resolve crosstalk, we propose that photons fed into a fiber can be degraded by offsetting the fiber from disc center of the bright star. Thus the bright and faint sources can be observed in the same plate without crosstalk contamination. We test the feasibility of the program with the test observation of LAMOST, and the results show that our program is consistent with the expectation.
\end{abstract}

Keywords. surveys, telescopes, spectrographs

\section{The algorithm of offsetting}

The atmospheric seeing: seeing $=2 \sqrt{2 \ln 2 r_{0}}$

The dynamic fiber efficiency of Gaussian point-like PSF:

$$
\eta=\iint_{(x-c)^{2}+y^{2}=r_{c}^{2}} \frac{1}{2 \pi r^{2}} e^{\frac{-\left(x^{2}+y^{2}\right)}{2 r_{0}^{2}}} d x d y
$$

Where $(x-c)^{2}+y^{2}=r_{c}^{2}$ represents the coupling part of the fiber head and star spot on the focal plane, $r_{c}$ stands for the fiber radius.

Seeing can be measured from the Guide star CCD camera, and so the efficiency with no offset, $\eta_{0}$, can be obtained. Considering a bright star $M^{\star}$, the actual light fed into the fiber decreases with the increasing offset $c$, if we want to degenerate it to the flux that a fainter star of magnitude $\mathrm{m}$ fed into the fiber with no offset in the same seeing condition, the offset c can be deduced from the following equation.

$$
\iint_{(\mathrm{x}-\mathrm{c})^{2}+\mathrm{y}^{2}=\mathrm{r}_{\mathrm{c}}^{2}} \frac{1}{2 \pi r^{2}} e^{\frac{-\left(x^{2}+y^{2}\right)}{2 r_{0}^{2}}} d x d y=\eta_{0} \times 10^{\frac{M^{\star}-m}{2.5}}
$$

\section{Result of the test}

Data were taken from test observation during the Pilot survey on Jan 2nd 2012. Targets were point sources selected from the SDSS database. The same targets were observed with two different fiber configurations with the bright stars $(r<15)$ in the second configuration offset to match the flux of star at magnitude 15 according to the previous formula, and leave the rest of the targets unchanged. The results show that, after offsetting the bright sources of $\mathrm{r} \sim 13-15$ to the flux level of $\mathrm{r} \sim 15$, the average $\mathrm{S} / \mathrm{N}$ of them are close to those of $\mathrm{r} \sim 15$. At the same time, observation without offset shows that the $\mathrm{S} / \mathrm{N}$ of $\mathrm{r} \sim 13-15$ stars are $30 \%$ larger than those of $\mathrm{r} \sim 15$. Therefore, the offset method works and can help fiber spectroscopic telescopes such as LAMOST to have a larger dynamic range in the same observation, and thus help to reduce the number of unused fibers when the targets are not dense enough. 